Friedrich L. Sell

\title{
Flexibles Inflationsziel für die Geldpolitik: nur neuer Wein in alten Schläuchen?
}

\begin{abstract}
Mit altgedienten Sprachbildern sollte man sparsam umgehen. Und doch: Die von Jerome Powell, dem Chef der Federal Reserve (Fed), unlängst vorgeschlagene zukünftige Zielzone für Inflationsraten, möglicherweise verbunden mit einer Anhebung der durchschnittlichen, von der Fed angestrebten Preissteigerungsrate, erinnert stark an ein von der Deutschen Bundesbank in den 1990er Jahren durchaus erfolgreich praktiziertes Modell. Damals stand allerdings nicht die Inflationsrate selbst, sondern die Wachstumsrate der Geldmenge im Fokus.
\end{abstract}

Zahlreiche Medien berichteten bereits Ende Juli 2020 davon: die Ankündigung von Jerome Powell, dass künftig in den USA „die Preissteigerung für eine Weile höher als zwei Prozent liegen [darf], wenn sie sich zuvor für einen längeren Zeitraum darunter bewegt hat" (Wiebe, 2020). Dieses Thema beschäftigt auch die EZB, mindestens seit dem Sommer des Jahres 2019: „Es gehe unter anderem um die Frage, ob das derzeitige Preisziel für die Zeit nach der Krise noch angemessen ist. [Der ehemalige] EZB-Präsident Mario Draghi bevorzuge einen „symmetrischen Ansatz“. Dabei hätte die Notenbank einen größeren Spielraum und könnte über einen längeren Zeitraum eine Abweichung vom Zielwert von zwei Prozent nach oben oder unten akzeptieren“ (NTV, 2019). Die Idee von Jerome Powell, aber eben auch von Mario Draghi, verschafft der Geldpolitik eine größere Flexibilität, denn sie muss nicht, wie bisher, bei überschäumender (abflauender) Konjunktur sofort mit Zinserhöhungen (Zinssenkungen) reagieren. Im Gegenteil: Sollte, nach einer längeren Schwächephase (Boomphase), die Inflation (wieder) anziehen (abfallen), so könnten die Leitzinsen dennoch weiter niedrig (hoch) bleiben, um die Stabilität des Wirtschaftswachstums abzusichern. „Der flexiblere Ansatz würde bedeuten, dass es nicht automatisch zu Zinserhöhungen käme, wenn wir bei zwei Prozent ange-

(c) Der/die Autor:in(nen) 2021. Open Access: Dieser Artikel wird unter der Creative Commons Namensnennung 4.0 International Lizenz (https:// creativecommons.org/licenses/by/4.0/deed.de) veröffentlicht.

Open Access wird durch die ZBW - Leibniz-Informationszentrum Wirtschaft gefördert.

Prof. em. Dr. Friedrich L. Sell lehrte VWL, insbesondere Makroökonomik und Wirtschaftspolitik, an der Universität der Bundeswehr München, Neubiberg. langt sind, sagte Devisenexpertin Thu Lan Nguyen von der Commerzbank“ (NTV, 2019).

Nun sind diese Formulierungen allesamt noch ziemlich vage. Für eine konkrete Ausgestaltung des Vorschlags braucht es wesentlich genauere Angaben. Immerhin hat sich EZB-Ratsmitglied Eberhard Nowotny etwas deutlicher als andere bisher aus dem Fenster gelehnt: Er sei dafür, das Zwei-Prozent-Ziel zu erhalten, „aber mit einem Korridor von 0,5 oder einem Prozent auf oder ab“ (NTV, 2019). Mit solcherlei Definition auf der Zielebene ist aber immer noch die Instrumentenebene unzureichend beschrieben. Wenn nämlich von $2 \%$ Preissteigerungsrate ${ }^{1}$ als dem angestrebten Durchschnitt die Rede ist, stellt sich zumindest die Frage, „welche vergangenen Zeiträume innerhalb welcher späteren Periode ausgeglichen werden sollen“ (Wiebe, 2020). Dabei spielt nicht nur die Länge der (vergangenen und zukünftigen) Zeiträume eine Rolle, sondern auch, ob strikte Symmetrie derselben angestrebt wird: Soll etwa die Zeitperiode der Überschreitungen ziemlich exakt derjenigen der Unterschreitungen entsprechen?

Jerome Powell präsentierte in Jackson Hole im Juli 2020 auch seine Sichtweise der neuen Instrumentenebene. Während die Steuerung des Zinsniveaus die erste Instrumentenebene der Fed bleibt, soll auf einer zweiten Ebene versucht werden, die Inflationserwartungen schon durch die Existenz eines Korridors zu stabilisieren. Wenn beispielsweise eine lang anhaltende, deutlich zu niedrige Preissteigerungsrate (Wiebel, 2020) die Inflationserwartungen zu drücken droht, eröffnet der Korridor den Marktteilnehmenden die Perspektive, dass künftige Zielüberschreitungen toleriert werden. Damit ließe sich ein Abgleiten der Inflationserwartungen

1 Dabei ist, wie Volker Wieland (2020) vom Sachverständigenrat zur Begutachtung der gesamtwirtschaftlichen Entwicklung zu Recht hinweist, noch nicht einmal klar, ob der von der EZB verwendete „Harmonisierte Verbraucherpreisindex" (HVPI) das geeignete Maß darstellt, um die Inflation in der Eurozone zu messen. Er bevorzugt, aus guten (hier nicht weiter diskutierten) Gründen, den BIP-Deflator. 
nach unten tendenziell stoppen. Im umgekehrten Fall verhindert der Korridor ein Wegdriften der Inflationserwartungen nach oben. Die Stabilisierung der Inflationserwartungen ist wiederum in diesem Konzept eine Schlüsselaufgabe oder ein Zwischenziel moderner Notenbanken, um die Inflation selbst als Endziel im Griff zu haben und zu behalten.

Allerdings funktioniert ein solcher Plan auch nur dann (gut), wenn bestimmte Voraussetzungen geklärt sind: Wenn z. B. eine Inflationsrate von $2 \%$ im Durchschnitt angestrebt wird und die oben skizzierte Symmetrie-Lösung (etwa zwei Perioden Vergangenheit und zwei Perioden Zukunft) zur Anwendung kommen soll, sind ganz verschiedene Szenarien denkbar: Nehmen wir im ersten Fall an, dass in der Vergangenheit Inflationsraten von $-1 \%$ und $+1 \%$ (Mittelwert 0\%) eingetreten sind. Dann könnte man sich für die Zukunft Inflationsraten von 1,5\% und von 6,5\% „leisten“ (Mittelwert $4 \%$ ). Denn in der Zusammenschau (der Mittelwert von $4 \%$ und $0 \%$ ist $2 \%$ ) könnte die Notenbank darauf verweisen, „im Durchschnitt“ (also über vier Perioden hinweg) eine Inflationsrate von $2 \%$ erreicht zu haben. In einem zweiten Szenario sollen Inflationsraten von $-0,5 \%$ und $+0,5 \%$ (Mittelwert $0 \%$ ) in der Vergangenheit und von 3\% bzw. $5 \%$ (Mittelwert $4 \%$ ) in der Zukunft gelten. Auch hier könnte die Notenbank guten Gewissens „Vollzug“ melden. Aber würden die privaten Haushalte sich in beiden Szenarien identisch/ähnlich verhalten? Wohl kaum! Das liegt daran, dass in beiden Szenarien deutlich verschiedene Extremwerte bzw. Varianzen vorliegen. Die Spreizung im ersten Szenario ist mit 7,5\% sehr groß, im zweiten dagegen mit 5,5\% deutlich niedriger. Je breiter der Korridor, desto weniger wird dieser vom Publikum für bindend gehalten. Um ihre Reputation zu erhalten, müsste die Notenbank, wie im Vorschlag von Nowotny, den Korridor ziemlich eng zuschneiden, ansonsten ist er wenig glaubwürdig. ${ }^{2}$ Prüfen wir zum Schluss den von Nowotny vorgeschlagenen Korridor anhand eines Beispiels, das die zu erwartenden Effekte verdeutlicht: In diesem dritten Szenario mit einem engen Korridor von plus/minus $1 \%$, sollen beispielsweise Inflationsraten von $1 \%$ und 1,5\% (Mittelwert 1,25\%) in der Vergangenheit und von $3 \%$ bzw. 2,5\% (Mittelwert 2,75\%) in der Zukunft gelten. Auch hier könnte die Notenbank darauf verweisen, dass sie (der Mittelwert von 1,25\% und 2,75\% ist 2\%), „im Durchschnitt“ (also über alle vier Perioden hinweg) eine Inflationsrate von $2 \%$ erreicht hat. Aber: Je enger der Korridor ist, desto eher sind auch Zielverletzungen denk-

2 Ein Beleg dafür ist die Entwicklung der Wechselkurszielzonen im Rahmen des Europäischen Währungssystems (EWS) I: Nach den erfolgreichen spekulativen Attacken gegen das Pfund Sterling und andere Währungen am Anfang der 1990er Jahre wusste man sich nur noch mit einer deutlichen Ausweitung des Paritätengitters zu behelfen. Das Thema der Härtung der europäischen Wechselkurs-Zielzone in Richtung Fixkurssystem war damit zunächst obsolet geworden. Erst die späteren Maastricht-Kriterien erzwangen eine geringere Volatilität in den bilateralen Wechselkursen. Vgl. dazu Giavazzi und Pagano (1988). bar, wenn nicht sogar wahrscheinlich: Auch dann leidet die Glaubwürdigkeit der Notenbank. Ein echtes Dilemma.

Anders als bisher angenommen, kann die Notenbank auch eine asymmetrische Lösung wählen. Dann gilt: Je unsymmetrischer die Verteilung zulasten der Vergangenheit und zugunsten der Zukunft - je mehr Zeit sich demnach die Geldpolitik dafür gibt, die Zielverfehlungen der Vergangenheit in der Zukunft zu korrigieren -, desto geringer ist die Bindungswirkung des Korridors im Hinblick auf die angestrebte Verankerung der Inflationserwartungen. Dasselbe gilt für die Dauer bzw. für die Zahl der Korrekturperioden insgesamt: Eine Notenbank macht sich unglaubwürdig, wenn sie das Erreichen eines Durchschnittsziels für die Inflationsrate verspricht, allerdings nur, wenn man das Ganze über viele, viele Jahre hinweg betrachtet. Gesucht wird also ein nach Möglichkeit symmetrischer Korridor, der eine optimale Breite/Spreizung in Verbindung mit einem maßvollen Zeithorizont verspricht. Nicht gerade ein Kinderspiel, auch für versierte Zentralbanker:innen nicht.

Das ist aber noch längst nicht alles: So darf z. B. nicht irgendeine, sondern es sollte eine ganz bestimmte Form der Erwartungsbildung im privaten Sektor vorliegen. Dabei handelt es sich weder um adaptive noch um rationale Erwartungen, also um diejenigen Erwartungsbildungshypothesen die in den meisten makroökonomischen Modellen (und damit auch in den Research-Abteilungen der großen Notenbanken) zur Anwendung kommen. Im besten Fall müssen „Confident expectations“ vorliegen. ${ }^{3}$ Diese wurden im Zusammenhang mit der Geldmengenstrategie der Deutschen Bundesbank (1948 bis 1998) erst im Jahr 1998 theoretisch entwickelt (Maaß und Sell, 1998), wenngleich sich der private Sektor unter dem Regime der Deutschen Bundesbank schon lange zuvor entsprechend verhalten haben dürfte. ${ }^{4}$

\section{Das Zielzonenkonzept der Deutschen Bundesbank}

Die Deutsche Bundesbank gab seit 1974 vielen Ländern in der Weltwirtschaft ein wichtiges Vorbild im Rahmen ih-

3 Unter "confident expectations“ versteht man, dass Erwartungsirrtümer im Hinblick auf die Inflationsrate zu einer Korrektur der Inflationserwartungen führen und zwar zu einer, die sich invers zur adaptiven Erwartungshypothese verhält: Während nämlich bei adaptiven Erwartungen bei Überraschungsinflation die Inflationserwartungen zunehmen, werden Wirtschaftssubjekte mit "confident expectations“ gerade umgekehrt annehmen, dass die (vorübergehend) erhöhte Inflationsrate in Zukunft nach unten korrigiert wird. Wenn nämlich das Überschreiten des Inflationsziels nur als transitorisch angesehen wird, rechnen die Wirtschaftssubjekte vernünftigerweise mit einem zukünftigen Unterschreiten des Inflationsziels, denn (nur) so kann die von der Notenbank angestrebte Inflationsrate im Durchschnitt erreicht werden.

4 Ein ähnliches „Schicksal“ erfuhr das EWS I, das seit 1978 bis 1998 mehr oder weniger stabil als Wechselkurszielzone funktionierte und dessen Existenz- und Stabilitätsbeweis erst im Jahr 1991 von Pau Krugman gewissermaßen „nachgeliefert“ wurde (Krugman, 1991). 
rer Inflationsbekämpfung ab, auch wenn sie, aus heutiger Sicht, damit nicht ganz so erfolgreich wie die EZB 1999 bis 2009 gewesen ist. Bis 1998 setzte sie sich ein Ziel für das jährliche Wachstum der Geldmenge. Wie wir heute wissen, hat sie dieses - auch bei Formulierung eines Zielkorridors für M3 - ebenso häufig verfehlt wie erreicht. Trotzdem hat damals niemand im In- oder Ausland ihren außerordentlichen Erfolg bei der Erhaltung der Preisniveaustabilität in Frage gestellt. Daher empfiehlt es sich, das von der Deutschen Bundesbank sehr erfolgreich angewendete Konzept eines monetären Zielkorridors noch einmal vorzustellen.

Im Konzept eines monetären Zielkorridors war es typischerweise und ganz bewusst so, dass der von der Zentralbank angekündigte Korridor für die Entwicklung des Geldangebots nicht immer eingehalten wurde. Das ist bereits ein wesentlicher Unterschied zur Nowotny-Konstruktion von oben. Vielmehr - das ist wiederum auch die Erfahrung mit der Deutschen Bundesbank - wurde er von den monetären Instanzen immer wieder einmal über- oder auch unterschritten (vgl. Abbildung 1). Die Bundesbank hat bis 1998 dabei aber nie ihre langfristige Zielsetzung - nämlich die Erhaltung von Preisniveaustabilität - aus den Augen verloren. ${ }^{5}$ Sogenannte transitorische Zielverletzungen - etwa, um externe Schocks zu akkommodieren - wurden in diesem Konzept so lange als vertretbar angesehen, wie sie im Privatsektor als nur vorübergehend eingeschätzt wurde und dort nicht zu einer Revision der mittel- und langfristigen Inflationserwartungen führte. Es war also keineswegs der Korridor allein, sondern die Überzeugung, dass sogar ein vorübergehendes Verlassen des Korridors nicht „schlimm“ sei, was die Inflationserwartungen stabil hielt. Eine Destabilisierung der Inflationserwartungen musste allerdings unbedingt vermieden werden. Dann nämlich wäre nicht nur die Reputation der Zentralbank, sondern auch die von ihr anvisierte Inflationsrate selbst (De Grauwe, 1992) in Gefahr geraten. Für die Notenbank wurde es demnach zu einer allesentscheidenden Frage, ob sie in der Lage war, eine mögliche (nachteilige) Änderung der Inflationserwartungen im Privatsektor zu verhindern, auch wenn sie selbst von ihren eigenen angekündigten Zielen vorübergehend abwich. ${ }^{6}$ Glaubwürdigkeit und Transparenz mussten hierfür gegeben sein, genau das ist der Deutschen Bundesbank damals gelungen: „monetary targeting provides a means of transparently and credibly communicating the relati-

5 „In any event, the Bundesbank was clearly allowing its short-term monetary policy to miss the targets in pursuit of the longerterm goal“. Zitat aus Mishkin und Posen (1997, 28).

6 Vgl. Maaß und Sell (1995). Nach Frankel und Hardouvelis (1985) liefern die Preisveränderungen von zentralen Rohstoffen, die direkt im Anschluss an Abweichungen der Notenbank von ihren eigenen Zielvorgaben auftreten, dieser die Information, ob im Privatsektor mit einer anschließenden Korrektur - zurück zum bisherigen geldpolitischen Kurs - gerechnet wird oder ob dort neue Zielvorgaben der Währungsbehörden für die Zukunft vermutet werden.
Abbildung 1

Wachstumsrate der Geldmenge M3 und Zielkorridor (Target) der Deutschen Bundesbank (1991 bis 1997)

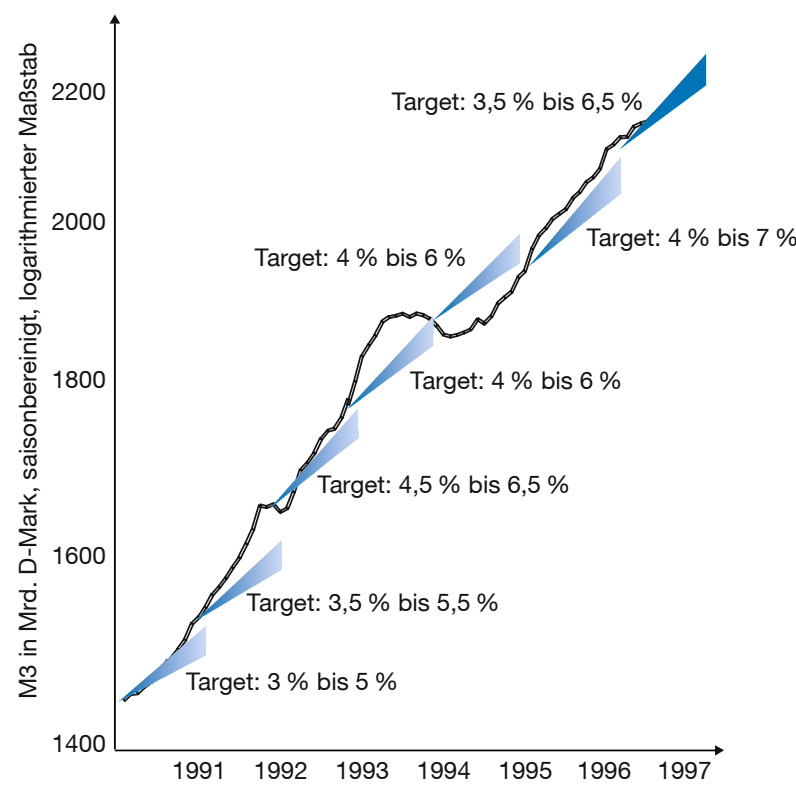

Quelle: Deutsche Bundesbank, Geschäftsbericht, verschiedene Jahrgänge; eigene Zusammenstellung.

onship between current developments and medium-term goals" (Mishkin und Posen, 1997, 21). In Abbildung 1 wird deutlich, dass das tatsächliche Geldmengenwachstum (M3) in den 1990er Jahren immer wieder aus den von der Deutschen Bundesbank festgelegten Korridoren ausgebrochen ist. Letztere waren nicht durchgehend konstant, sondern lagen zwischen (mindestens) $3 \%$ und (höchstens) $7 \%$. Gerade in den 1980er und in den 1990er Jahren galt die Deutsche Bundesbank als Hort der Preisstabilität.

Ihre Reputation können Zentralbanken demnach selbst dann behalten, wenn sie den privaten Sektor (sogar mehr als) einmal täuschen; dies ist immer dann möglich, wenn sie sich über (viele) lange Jahre hinweg erfolgreich um Preisniveaustabilität bemüht haben. ${ }^{7}$ Die Reputation einer Zentralbank ist nämlich selbst eine Funktion der Dauer der bisher von ihr „hergestellten“ Preisniveaustabilität (Maaß und Sell, 1998; Sell, 1999). Das Publikum erwartet unter diesen Bedingungen nach einem Überschreiten (Unterschreiten) des Geldmengenkorridors ein entsprechend kompensierendes Gegensteuern. Es liegen dann gewissermaßen „perverse“ adaptive Erwartungen vor, die man auch als „rationale Vertrauensseligkeit“ („confident expectations“) des Publikums interpretieren könnte. Eine spiel- bzw. zeitinkonsistenzthe-

7 „In short, as long as the underlying inflation goal was met over the medium term, the existence of the monetary targets rather than their precise funcionality was sufficient" (Mishkin und Posen, 1997, 27). 
oretische Fundierung dieser Erwartungshypothese findet sich bei Maaß und Sell (1998).

Im Detail basieren „confident expectations“ auf den folgenden Hypothesen (Maaß und Sell, 1998, 524):

1. Nicht ein einzelner, kurzfristiger Wert der Inflationsrate, sondern nur die langfristige durchschnittliche Inflationsrate ist ein sinnvoller Indikator für den Erfolg von Geldpolitik.

2. Kurzfristige Abweichungen der realisierten von der langfristigen Ziel-Inflationsrate (bzw. vom langfristigen Zielkorridor für die Inflationsrate) müssen früher oder später korrigiert werden. Dies geschieht am besten durch eine kompensierende diskretionäre Geldpolitik, welche die Reputation einer erfolgreichen Notenbank zu bewahren hilft.

3. Nur dann, wenn die langfristigen Ergebnisse der Geldpolitik von den beteiligten Akteur:innen gebilligt werden, kann die Reputation einer erfolgreichen Notenbank hoch bleiben oder werden.

Liegen solchermaßen "confident expectations" vor, so lässt sich zeigen, dass - bei unterstelltem Optimierungsverhalten der Notenbank in einer Barro-Lucas-SargentWallace-Welt ${ }^{8}$ - nach einer „price surprise“ schnell zu hoher Preisniveaustabilität zurückgekehrt werden kann, ${ }^{9}$ während rationale Erwartungen dagegen in eine anhaltend hohe Inflationsrate münden (vgl. Maaß und Sell, 1995; 1998). Ein solches Resultat belegt das (an dieser Stelle auch von der EZB geteilte) monetaristische Credo, wonach bereits eine Stabilisierung der Inflationserwartungen zur Dämpfung der Inflation selbst beiträgt. Zugleich wirft es die Frage auf, ob das von der neuklassischen Theorie behauptete Paradigma, wonach der private Sektor nach einer einmaligen Täuschung die Reputation der Zentralbank für lange Zeit ad acta legt, nicht dazu führt, dass sich die Wirtschaftssubjekte durch rationale Erwartungen gewissermaBen selbst bestrafen. ${ }^{10}$

8 In einer solchen Welt gilt die sogenannte „Lucas-Kritik“, wonach bei Kurswechseln in der Geld- oder in der Finanzpolitik aus der Sicht der handelnden Instanzen mit einem entsprechenden (und zwar unerwünschten) Anpassungsverhalten des privaten Sektors (Haushalte, Unternehmen) gerechnet werden muss. Wenn aber kurzfristige Zielverletzungen der Politik vom privaten Sektor nur als solche, nicht aber als Symptome eines wirklichen Kurswechsels gedeutet werden, können diese (unerwünschten) Anpassungsreaktionen ausbleiben.

9 Auch Laskar (1994) plädierte 1994 für einen Zielkorridor der Geldpolitik als Hebel, um zeit(in)konsistentem Verhalten von Notenbanken entgegenzuwirken.

10 Der Ordnung halber sei eingeräumt, dass die Literatur zum zeitinkonsistenten Verhalten von Notenbanken schon früh auf den sogenannten „Grimm-Trigger-Mechanismus“ hingewiesen hat. Danach können die zukünftigen Verluste des Reputationsverlustes bzw. die entgangenen Nutzen aus Kooperation - bei niedriger Zeitpräferenzrate des privaten Sektors - so hoch ausfallen, dass sich Täuschungsverhalten für die handelnde Notenbank nicht wirklich lohnt (Currie und Levine, 1993).

\section{Gemeinsamkeiten und Unterschiede}

Gemeinsam ist der Deutschen Bundesbank und den groBen Notenbanken dieser Tage ganz gewiss die Erkenntnis, dass eine Kontrolle der Inflationsrate entscheidend davon abhängt, die Inflationserwartungen des privaten Sektors stabil halten zu können. Diesem Kriterium muss sich jedes noch so subtile Arrangement der Geldpolitik, also auch der Powell-Vorschlag unterwerfen. Trotz der absehbaren Konstruktionsunterschiede ist erkennbar, dass der Deutschen Bundesbank dabei nach wie vor eine frühe Vorreiter- und auch späte Vorbildrolle zukommt. Ein wichtiger Unterschied zur Politik der Deutschen Bundebank besteht zweifellos darin, dass die heutigen modernen Notenbanken, wie die EZB und die Fed, es in der Gegenwart mit einem deflationären, wenn nicht schon teilweise deflatorischen Umfeld zu tun haben. Gelegenheit zum „Unterschreiten“ ihrer monetären Expansionsrate wird innen zurzeit gewissermaßen kaum noch geboten. Studien zur Effektivität/Effizienz der Geldpolitik in den großen Industrienationen würden zeigen, dass die beispiellose Ausweitung der Bilanzsummen/Zentralbankgeldmengen seit 2008 nur von (sehr) geringem Erfolg gekrönt waren, jedenfalls im Hinblick auf das Erreichen des von den Notenbanken selbst gesetzten Inflationsziels. ${ }^{11}$

Wenn es stimmt, dass die Reputation einer Zentralbank selbst eine Funktion der Dauer der bisher von ihr hergestellten Preisniveaustabilität ist, dann haben Fed und EZB, anders als früher die Deutsche Bundesbank, gegenwärtig ein Problem: Es ist innen gerade in den letzten zehn, elf Jahren nicht gelungen, ihr selbst gesetztes Inflationsziel überzeugend zu erreichen. Unter diesem Reputationsmangel würde auch jede zukünftige Korridorkonstruktion leiden. Ein harmonisches Zusammenspiel von Zielzone der Notenbank mit "confident expectations" des privaten Sektors ist daher deutlich weniger wahrscheinlich als im Falle der Deutschen Bundesbank. Während die Bundesbank sich bei ihrem Korridor auf den Einsatz eines Instruments (Zentralbank- bzw. Geldmenge) bezog, zielt der Powell-Vorschlag gewissermaBen auf den Erfolgsindikator für die Politik der Zentralbank schlechthin, nämlich auf die Inflationsrate selbst ab. Das ist insofern ein schwierigerer Job, als es Notenbanken wesentlich leichter fallen dürfte, die Expansion oder Kontraktion ihrer eigenen Bilanzsummen zu kontrollieren als die Höhe der Inflationsrate. Bekanntlich liegen zahlreiche Einflussgrößen für diese Variable nach wie vor mehr oder weniger außerhalb des Wirkungsgrads von Notenbanken (Terms of Trade, Wechselkurs, ausländisches Zinsniveau etc.). Das Konzept

11 „Die Notenbanken haben offensichtlich die Kontrolle über die Entwicklung des Preisniveaus verloren." Zitat aus Rürup $(2020,12)$. Stattdessen sind die Vermögenspreise durch die Decke gegangen, verbunden mit erheblichen regressiven Effekten auf die Vermögensverteilung. 
der Deutschen Bundesbank wies im Vergleich zum PowellVorschlag einen Freiheitsgrad mehr auf, waren doch dort transitorische Ausbrüche aus dem Zielkorridor durchaus tolerabel. In der Nowotny-Version des Powell-Vorschlags gibt es diesen Freiheitsgrad (noch) nicht. Es bietet sich an, entweder den Korridor etwas weiter zu schneiden als im Nowotny-Ansatz oder aber Ausbrüche aus dem engen Korridor von vornherein in die Kompensationspolitik mit einzubeziehen.

Ein grundsätzliches Problem können allerdings auch die ausgetüfteltesten Vorschläge nicht überwinden: Wir wissen nicht, ob es in Zukunft noch Inflation wie zu Zeiten der Deutschen Bundesbank, also bis Ende der 1990er Jahre, geben wird. Das würde dem expansiven Teil der Kompensationsstrategie von Korridorkonstrukten eine klare Schranke setzen. Es gibt genügend Argumente, die auch weiterhin anhaltend niedrige Inflationsraten, wie seit 2009, nahelegen (Rürup, 2020, 12): Die Globalisierung hat zu einer Wettbewerbsverdichtung geführt, die den Preiserhöhungsspielraum der Unternehmen einschränkt. Die Digitalisierung hat wiederum die Produktionskosten gesenkt. Lohn-Preisspiralen, die von den Notenbanken besonders in den 1970er bis 1990er Jahren gefürchtet waren, haben an Bedeutung und Relevanz eingebüßt, seitdem die Gewerkschaften in vielen Ländern, gerade auch in der Eurozone, Mitglieder und Verhandlungsmacht zunehmend verloren haben (Öllinger und Sell, 2017).

\section{Fazit}

Ein Blick in die Vergangenheit der Deutschen Bundesbank zeigt, dass sie mit dem Konzept eines Zielkorridors für die Geldmenge nicht nur ein operationales Zwischenziel der Geldpolitik formuliert, sondern damit auch in besonderer Weise der Erwartungsstruktur des Publikums entsprochen hat. Durch den langfristigen Erfolg an der Inflationsfront geriet sie bei Zielverletzungen des Geldmengenkorridors eigentlich nie in ernsthafte Gefahr, ihre Reputation einzubüßen. Ein ähnlich motivierter Zielkorridor für die Inflationsrate könnte nicht nur für die Fed, sondern auch für die EZB zu einem wichtigen Element ihrer Geldpolitik werden. Dabei sollte die EZB - gerade wegen des Reputationsmangels im Hinblick auf das Einhalten des eigenen Inflationsziels - bewusst einen direkten Bezug zur Politik der Deutschen Bundesbank in der Vergangenheit herstellen, um so gewissermaßen Reputation für ihren künftigen Korridor zu borgen, die sie selbst noch in den kommenden Jahren beim Publikum erwerben muss. Diesen Startvorteil hat die Fed nicht. Die im Titel dieses Beitrags eher rhetorisch gestellte Frage nach dem „neuen Wein in alten Schläuchen“ lässt sich bejahen, das führt aber nicht zu einer Fundamentalkritik am Powell-Vorschlag. Mit einer klugen Ausarbeitung im Detail kann damit die Qualität der gegenwärtigen europäischen und auch der US-amerikanischen Geldpolitik sogar noch deutlich verbessert werden.

\section{Literatur}

Currie, D. und P. Levine (1993), Rules, Reputation and Macroeconomic Policy Coordination, Cambridge University Press.

De Grauwe, P. (1992), The Economics of Monetary Integration.

Frankel, J. A. und G. A. Hardouvelis (1985), Commodity Prices, Money Surprises and Fed Credibility, Journal of Money, Credit, and Banking, 17(4), 425-438.

Giavazzi, F. und M. Pagano (1988), The Advantage of Tying One's Hands: EMS Discipline and Central Bank Credibility, European Economic Review, 32(5), 1055-1077.

Krugman, P. (1991), Target Zones and Exchange Rate Dynamics, Quarterly Journal of Economics, 106(3), 669-682.

Laskar, D. (1994), „Time inconsistency“ of the optimal monetary policy: a case for target zones, Centre d'Études Prospectives d'Économie Mathématique Appliquées à la Planification, Working Paper.

Maaß, H. und F. L. Sell (1995), Rohstoffpreise, Inflationserwartungen und internationale Politikkoordination, Dresdner Beiträge zur Volkswirtschaftslehre, Nr. 17.

Maaß, H. und F. L. Sell (1998), Confident Expectations, Rational Expectations and the Optimal Conduct of Monetary Policy, Economic Modelling, 5(4), 519-541.

Mishkin, F. S. und A. S. Posen (1997), Inflation Targeting: Lessons From Four Countries, Federal Reserve Bank of New York Economic Policy Review, 3(3), 9-110.

NTV (2019), EZB überprüft offenbar ihr Inflationsziel, 18. Juli, www.n-tv. de/wirtschaft/EZB-ueberprueft-offenbar-ihr-Inflationsziel-article21154364.html (28. Dezember 2020).

Öllinger, M. und F. L. Sell (2017), What determines union density? A political economy model of the labor market with empirical evidence in the context of European countries, Review of Economics and Finance, 10(4), 18-32.

Rürup, B. (2020), Der heimliche Gewinner der Pandemie, Handelsblatt, Nr. 221, 13. November, 12.

Sell, F. L. (1999), Zielzonen für die Wechselkurs- oder für die Geldpolitik?, List Forum für Wirtschafts- und Finanzpolitik, 25(1), 1-11.

Wiebe, F. (2020), Neuer Spielraum. Der Chef der US-Notenbank Fed kündigt ein flexibleres Inflationsziel an. Für die Anpassung präsentiert er eine subtile Rechtfertigung, Handelsblatt, 28. Juli, 32.

Wieland, V. (2020), Inflation ist nicht gleich Inflation, Handelsblatt, Nr. 220, 12. November, 8 .

Title: Flexible Inflation Target for Monetary Policy

Abstract: This paper analyses the Federal Reserve's Jerome Powell's proposal possibilities and limits made during the recent meeting of central bankers in Jackson Hole, Wyoming, USA. According to Powell, the Fed, as well as the ECB, might change its inflation target policy in the future. The idea is to construct a target zone for the inflation rate. This mechanism would allow the Fed to violate its own inflation target possibly more than $2 \%$ in the future - for certain periods, provided it is committed to compensate for these violations in subsequent periods. The goal, hence, would be to meet the inflation target only on average in each period. This article discusses problems of implementation, compares the plan with the monetary compensation policy of the old Deutsche Bundesbank and assesses its likely failure or success. 